\title{
Förderpreis Nephrologische Pflege 2015 Verleihung beim 37. AfnP Symposium am 28.11.2015
}

Den Förderpreis Nephrologische Pflege 2015 gewannen punktgleich 3 Bewerber: Sophie Stedtler mit dem Thema „Nephrologische Patienten im Krankenhaus - Optimierung der Betreuung“, Saskia Pavelcsik mit dem Thema „(Prä-)Dialysepflichtige Kinder im Alter von 6-8 Jahren - Entwicklung eines Schulungskonzepts“ sowie Joachim Strasser mit dem Thema „Palliative Versorgung von Dialysepatienten - Aktuelle Versorgungsstruktur im Landkreis Dillingen“.

Zum zehnten Mal war die AfnP e.V. dank der großzügigen Unterstützung des Georg Thieme Verlags in der Lage, den Förderpreis Nephrologische Pflege zu vergeben. Der Förderpreis Nephrologische Pflege wurde 2015 erstmalig an 3 Bewerber zu gleichen Teilen vergeben. Die 3 Preisträger erhielten jeweils ein Drittel des vom Georg Thieme Verlag gesponserten Preisgeldes in Höhe von je 500 Euro plus jeweils noch einen Bonus von je 100 Euro, da die Arbeiten eine besonders hohe Qualität aufwiesen. Außerdem erhielten die Preisträger jeweils einen Büchergutschein für das Thieme Sortiment über je 200 Euro. Den ersten Platz teilten sich folgende 3 Bewerber bzw. Beiträge:

- Sophie Stedtler (Abb. 1), Lutherstadt Eisleben, mit dem Thema „Nephrologische Patienten im Krankenhaus Optimierung der Betreuung“

- Saskia Paveleski (Abb. 1), Neustadt an der Weinstraße, mit dem Thema „(Prä-)Dialysepflichtige Kinder im Alter von 6-8 Jahren - Entwicklung eines Schulungskonzepts“
- Joachim Strasser (Abb. 1), Dillingen, mit dem Thema „Palliative Versorgung von Dialysepatienten - Aktuelle Versorgungsstruktur im Landkreis Dillingen“

\section{Hohe Qualität vieler Arbeiten}

Bemerkenswert ist, dass sich durch alle 3 Arbeiten in den einzelnen Bereichen vieles verändert hat und noch im Umbruch ist. Die Qualität der diesjährigen eingereichten Arbeiten war so hoch, dass die Redaktion plant, einige der besten Arbeiten im Laufe der Zeit in der Dialyse aktuell zu publizieren.

Im Jahr 2015 reichten so viele Einzelpersonen und Gruppen ihre Arbeit ein wie noch nie zuvor. Alle eingereichten Arbeiten erfüllten die Kriterien und kamen damit in die Bewertung. Die Bewertungskriterien und alle Informationen rund um den Pflegepreis sowie die Arbeiten der (vorigen) Preisträger finden Sie auf der AfnP-Homepage (www.afnp.de). Die Beiträge, die auf den Arbeiten der aktuellen Preisträger basieren, finden Sie in der vorliegenden Ausgabe der Dialyse aktuell.

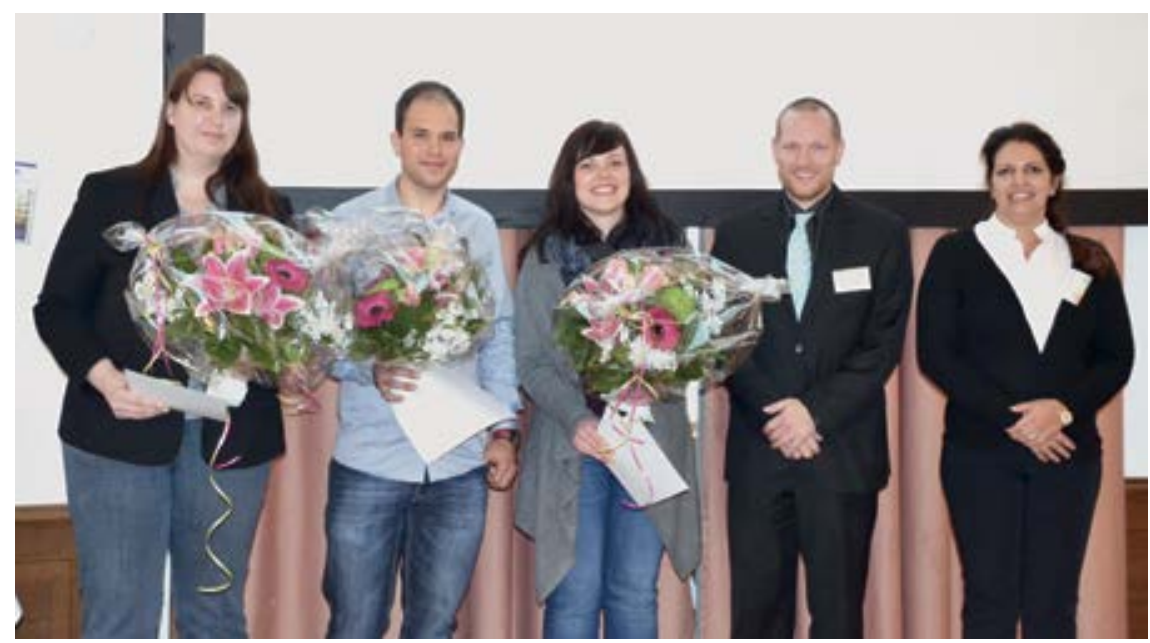

Abb. 1 Die 3 Preisträger und die Laudatoren nach der Preisvergabe (von links): Saskia Pavelcsik, Joachim Strasser, Sophie Stedtler, Christian Schäfer (Georg Thieme Verlag) und Marion Bundschu (AfnP).

\section{Themenspektrum war weit gefächert}

Auf den Platz hinter den Erstplatzierten kam mit nur 0,2 Punkten Unterschied Lidia Kowalska, Kempten, mit dem Beitrag „Ganzheitliche Pflege im Dialysezentrum - Wunschdenken oder Wirklichkeit?". Dies ist eine sehr gelungene Facharbeit mit hervorragenden Herleitungen und Begründungen. Die interessante Betrachtung ist absolut lesenswert. Weitere Themen waren:

- Hämodialyse oder Peritonealdialyse - Welches ist das bessere Dialyseverfahren für mich als älterer Patient? Die Aufklärung über Nierenersatzverfahren

- Nephrologische Pflege trifft geriatrische Pflege - Der dialysepflichtige Heimbewohner mit kognitiven Veränderungen

- Einführung eines systematischen Shunt-Monitorings bei veränderten Strukturen in der Dialyse

- Shuntpunktion - Quantität oder Qualität? Problem Arealpunktion

- Informationsbroschüre für medizinisches, nicht nephrologisches Klinikpersonal zum fachgerechten Umgang mit dem Dialysekatheter

- Behauptung, dass eine niedrige Dialysattemperatur eine effektivere Dialyse bewirkt

- Beugt das Umhüllen des Dialysators mit Aluminiumfolie während der CVVH einem Dialysatverlust des Patienten vor?

- Unterstützung der Krankheitsbewältigung chronisch niereninsuffizienter Menschen durch Ernährungsberatung

- Therapietreue bei Dialysepatienten im Zusammenhang mit Coping

\section{Fachjury}

Die Fachjury setzt sich wie folgt zusammen:

- 2 ärztliche Fachjuroren

- 2 pädagogische Leitungen von nephrologischen Fachschulen

- Diplom-Fachwirtin und nephrologische Fachschwester

- Master of Education

- $\quad$ ein Vertreter des Georg Thieme Verlags 
- $\quad$ ein Vertreter der Bayrischen $\mathrm{KH}-\mathrm{Ge}-$ sellschaft

Es ist trotz des Arbeitsaufwandes immer wieder spannend und sehr informativ, die Arbeiten zu lesen. Unsere qualifizierte Jury führt diese Aufgabe jedes Jahr ehrenamtlich durch - dafür ein besonderer Dank! Überreicht wurden die Preise von Christian Schäfer (Abb. 1), Georg Thieme Verlag, und von Marion Bundschu (Abb. 1), 1. Vorsitzende der AfnP e.V.

\section{Infos zum Förderpreis \\ Nephrologische Pflege}

Die Beiträge, die auf den oben erwähnten 3 Arbeiten basieren, finden Sie wie beschrieben in dieser Ausgabe der Dialyse aktuell. Zudem erhalten die Verfasser noch ein Autorenhonorar. Sie sehen, es gibt neben dem Preisgeld noch eine Menge anderer Anreize, Arbeiten für den Pflegepreis einzureichen. Dies könnte auch Sie dazu animieren, Beiträge zu verfassen und abzugeben. Reichen Sie Ihre Arbeiten für den Preis 2016 ein!

\section{Kriterien}

Das Themenspektrum ist weit gefächert und umfasst alle deutschsprachigen Arbeiten (auch aus A, CH, L etc.) aus dem nephrologischen Bereich, wie die präterminale und terminale Phase mit den entsprechenden Nierenersatzverfahren sowie die Transplantation und verwandte Gebiete wie z.B. die Apheresetherapie.
Die Thematik sollte am besten einen Bezug zur nephrologischen Pflege haben und kann ein Bericht über ein praxisrelevantes Projekt sein, das Sie in Ihrem beruflichen Umfeld umgesetzt haben oder mit dem Sie bereits erste Erfahrungen im Alltag sammeln konnten. Diese Hinweise sollen Ihnen Unterstützung geben, damit Sie Ihre Arbeit mit der Aussicht auf Erfolg erstellen können. Bitte beachten Sie außerdem, wenn Sie Ihre Arbeit einreichen möchten:

- Arbeiten dürfen als Einzel- oder Gruppenarbeiten eingereicht werden. - Wichtig ist uns als Jury der Bezug zur nephrologischen Pflege. Auch Themen, die übergeordnet interessant sind, sind uns willkommen. Jedoch

\section{Steckbriefe der 3 Preisträger und Begründungen für die Themenwahl}

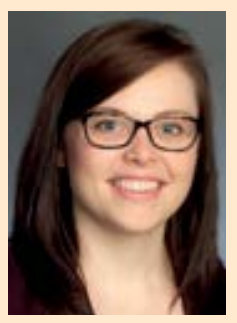

Sophie Stedtler

geboren 1990, wohnhaft Lutherstadt Eisleben

Beruflicher

Werdegang

- 2011: Examen Gesundheits- und Krankenpflegerin

- 2011-2012: Intensivtherapiestation der Poliklinik für Herz- und Thoraxchirurgie Universitätsklinikum Halle (Saale)

- 2012-heute: Dialysepraxis Dr. Kresse \& Dr. Langer, Lutherstadt Eisleben

- 2013-2015: Fachausbildung Nephrologie und Dialyse Weiterbildungsstätte Ulm

- 2015: Praxisanleiter Weiterbildungsstätte Ulm

Erster Kontakt zur AfnP

- während der Fachausbildung durch Marion Bundschu

Der Rest meiner Zeit gehört ..

- ... meiner Familie, meinen Freunden, dem Fahrradfahren und der Handarbeit/dem Basteln.

Wahl des Themas

Mein Beitrag beschäftigt sich mit den alltäglichen Problematiken, die bei der Pflege mit nephrologischen Patienten auftreten. Vor allem während eines Krankenhausaufenthaltes werden diese besonders verstärkt. Unsere Dialysepraxis kooperiert mit dem städtischen Krankenhaus, welches keine nephrologische Station oder Dialyse besitzt. Demzufolge initiierte ich im Rahmen meiner Fachausbildung ein Projekt, um die Zusammenarbeit und die Pflegequalität zu verbessern. Ich habe mich für dieses Thema entschieden, um reibungslose Arbeitsabläufe für den Alltag zu schaffen und auf unser Fachgebiet aufmerksam zu machen.

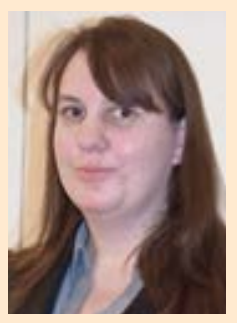

\section{Saskia Pavelcsik}

wohnhaft in Neustadt an der Weinstraße

\section{Beruflicher Werdegang}

- Gesundheits- und Krankenpflegerin (generalisiert), Universitätsmedizin Mainz

- 2012 Bachelor of Arts Pflege, HAW Hamburg

- derzeit Master Thesis im Studiengang „Pflege Advanced Practice Nursing“, Frankfurt University of Applied Sciences

- seit 2015 berufsbegleitendes M.A.-Studium „Pädagogik für Pflege- und Gesundheitsberufe“, Universität Kassel
- seit 2015 berufsbegleitendes M.Sc.-Studium „Integrative Health Science“, Högskolan Kristianstad (Schweden)

Wahl des Themas

Im Rahmen meines Bachelor-Studiums habe ich im Sommer 2011 ein Praktikum in der Kinderdialyse am UKE Hamburg absolviert - genau zur Zeit des großen EHEC-Ausbruchs. Es mussten deutlich mehr Kinder als sonst dialysiert werden und mich erschreckte sehr, dass die Kinder kaum zusammenhängende und nur wenig kindgerechte Informationen zu ihrer Erkrankung und zu ihren nun notwendigen neuen Verhaltensweisen erhielten. Dies war für mich Anlass genug, ein Schulungskonzept zu entwerfen und stetig weiterzuentwickeln.

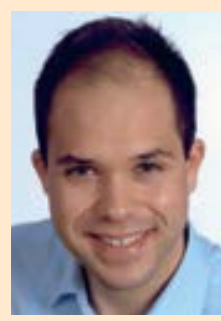

Joachim Strasser

geboren 1987, wohnhaft in Dillingen an der Donau

Beruflicher Werdegang

- $\quad$ 2004-2007: Ausbildung zum Gesundheits- und Krankenpfleger am Kreiskrankenhaus St. Elisabeth in Dillingen an der Donau

- 2007-2008: Gesundheits- und Krankenpfleger in der Zusamklinik der Deutschen Rentenversicherung Schwaben

- seit 2008: tätig im KfH Dillingen an der Donau

- 2015: Qualifikation zum Fachpfleger für Nephrologie an der Weiterbildungsstädte Ulm

- 2015: Qualifikation zum Praxisanleiter an der Weiterbildungsstädte Ulm

Erster Kontakt zur AfnP:

- über Marion Bundschu

- Mitglied seit 2015

Der Rest meiner Zeit gehört ...

... meinen Hobbys, meiner Familie und meinen Freunden.

Wahl des Themas:

In meiner täglichen Arbeit empfand ich die Versorgung unserer multimorbiden palliativen Patienten außerhalb der Dialyse als sehr schwierig. Dies gab mir den Anlass, diese Versorgungsstruktur näher zu beleuchten. In unserer ländlichen Region stecken viele Strukturen hinsichtlich der palliativen Versorgung noch in den Kinderschuhen und für die Angehörigen ist es oft schwierig, einen Ansprechpartner für den ersten Kontakt zu finden. Die Frage, wann mit der palliativen Versorgung begonnen werden soll, war für mich ebenfalls unklar. Um die aktuelle Situation in unserem Landkreis darzustellen und diese Frage beantworten zu können, habe ich die Facharbeit verfasst. 
erwarten wir einen konkreten Transfer zu Ihrem Arbeitsfeld.

- Ihre eigene Erfahrung ist wichtig und notwendig. Sie kann die Grundlage zur Beschäftigung mit einem selbst gewählten Thema sein. Darüber hinaus sollten Sie jedoch reine Erfahrungsberichte vermeiden.

- Ihre Arbeit sollte sich durch eine deutliche Hinführung zum Thema und zielgerichteter Fragestellung auszeichnen. Eine (kurze) Zusammenfassung der Arbeit ist zu erstellen. Die Gliederung sollte in der Logik an dieser Eingrenzung des Themas orientiert sein. Das Thema muss in angemessener Tiefe behandelt sein und Antworten auf die Problemstellung zulassen. Es sollte möglich sein, daraufhin Konsequenzen abzuleiten und diese auch darzustellen.

- Ihr Thema sollte so aufgearbeitet sein, dass Regeln des wissenschaftlichen
Arbeitens erkennbar werden. Dazu finden Sie unten Literaturempfehlungen oder Sie nutzen andere Quellen, die Sie in dieser Hinsicht unterstützen könnten.

- Wir freuen uns über die Vielfalt - Sie können Literaturarbeiten, Projektarbeiten oder empirische Arbeiten einsenden.

- Neben den oben angegebenen Punkten achten wir auf die saubere, ordentliche Ausfertigung Ihrer Arbeit. Dazu gehören auch eine angemessene sprachliche Ausdrucksfähigkeit und die korrekte Benutzung der Fachsprache.

- Selbstverständlich akzeptieren wir keine Plagiate. Diese Arbeiten scheiden sofort aus.

- Die Arbeit darf bei keinem anderen Pflegepreis eingereicht sein. Sie wurde auch in der Vergangenheit mit noch keinem Pflegepreis ausgezeich- net und wurde bis jetzt noch nicht veröffentlicht.

\section{Weitere Infos}

Hilfreiche Literatur sind z.B. die Bücher von Stickel-Wolf und Wolf [1] und von Kruse [2]. Interessierte können entsprechende Einzel- oder Gruppenarbeiten bei der AfnP-Geschäftsstelle als WordDokument vorzugsweise per E-Mail, USBStick oder CD einreichen (AfnP-Geschäftsstelle, Käppelesweg 8, 89129 Langenau, info@afnp.de). Für den Förderpreis Nephrologische Pflege 2016 ist der Einsendeschluss der 01.08.2016.

Marion Bundschu, Ulm

\section{Literatur}

1 Stickel-Wolf C, Wolf J. Wissenschaftliches Arbeiten und Lerntechniken. Erfolgreich studieren - gewusst wie! 6. Aufl. Wiesbaden: Gabler; 2011

2 Kruse O. Keine Angst vor dem leeren Blatt. Ohne Schreibblockaden durchs Studium. 12. Aufl. Frankfurt am Main: Campus; 2007 